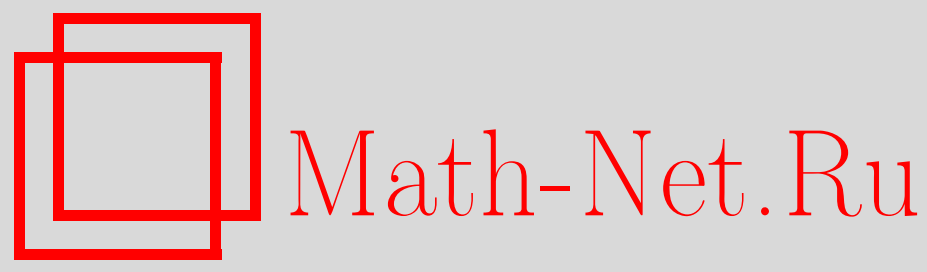

Z. G. Su, On Central Limit Theorems for Vector Random Measures and Measure-Valued Processes, Теория вероятн. и ее примен., 2001, том 46, выпуск 3, 513-534

DOI: https://doi.org/10.4213/tvp3899

Использование Общероссийского математического портала Math-Net.Ru подразумевает, что вы прочитали и согласны с пользовательским соглашением

http://www . mathnet.ru/rus/agreement

Параметры загрузки:

IP : 54.196 .121 .252

26 апреля 2023 г., 05:57:13

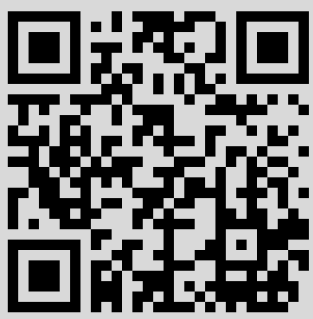




\title{
ON CENTRAL LIMIT THEOREMS FOR VECTOR RANDOM MEASURES AND MEASURE-VALUED PROCESSES ${ }^{1)}$
}

\begin{abstract}
Пусть $B$ - сепарабельное банахово пространство. Предположим, что $\left(F, F_{i}, i \geqslant 1\right)$ - последовательность независимых, одинаково распределенных, симметричных и независимо разбросанных случайных мер со значениями в $B$. Мы устанавливаем центральную предельную теорему для $Y_{n}=\frac{1}{\sqrt{n}} \sum_{i=1}^{n} F_{i}$, рассматривая случайные линейные функционалы на пространствах распределений Шварца. В тех же рамках исследуется центральная предельная теорема для мерозначных процессов $Z_{n}(t)=\frac{1}{\sqrt{n}} \sum_{i=1}^{n} X_{i} \delta_{B_{i}(t)}$, $t \in[0,1]$, где $\left(X, X_{i}, i \geqslant 1\right)$ - последовательность независимых, одинаково распределенных, симметричных случайных векторов со значениями в $B$ и $\left(B, B_{i}, i \geqslant 1\right)$ - последовательность независимых стандартных броуновских движений на $[0,1]$, не зависящих от $\left(X, X_{i}, i \geqslant 1\right)$. Наши основные результаты, касающиеся $Y_{n}$, отличаются от результатов [8] тем, что мы рассматриваем $F$ в целом, тогда как утверждения, связанные с $Z_{n}$, являются обобщением [7] на случайные взвешенные массы.
\end{abstract}

Ключевые слова и фразы: центральная предельная теорема, гауссовский процесс, случайная векторная мера, пространства Шварца.

1. Introduction. The concept of measure-valued process has its origin at the evolution in time of the population. Consider a population each of whose individuals is represented by its state $x \in \mathbf{R}$. Assume that the state of the population is completely described by the states of the individuals $\left\{x_{i}, i \in I(t)\right\}$, where $I(t)$ is the set of living individuals at time $t$. A wellestablished representation for such a population can be obtained by setting

$$
X(t)=\sum_{i \in I(t)} \varepsilon \delta_{x_{i}}
$$

where $\delta_{x}$ denotes the Dirac measure at point $x$ and $\varepsilon$ is a normalizing factor which may be one or represent the mass of each particle if the individuals are particles.

${ }^{*}$ Department of Mathematics, Hangzhou University, 310028, China; e-mail: zgsu@mail.hz.zj.cn

1) Research supported by the Foundations of National Natural Science of China and Zhejiang Province. 
In many situations one is led to consider sequences $X_{n}(\cdot)$ of this type of processes and to study the limit of their laws. We refer to Itô [5], Mitoma [7], and Walsh [10]. Let $\left\{B_{k}(t), t \in[0,1]\right\}, k=1,2, \ldots$, be a sequence of independent 1-dimensional Brownian motions with $B_{k}(0)=0$ for each $k \geqslant 1$. Define a sequence of measure-valued processes $X_{n}(t, \cdot)$ as follows.

For a Borel subset $A \in \mathscr{B}(\mathbf{R})$

$$
N_{n}(t, A)=\sum_{k=1}^{n} \delta_{B_{k}(t)}(A)
$$

and

$$
\begin{aligned}
X_{n}(t, A) & =\frac{1}{\sqrt{n}}\left(N_{n}(t, A)-\mathbf{E} N_{n}(t, A)\right) \\
& =\frac{1}{\sqrt{n}}\left(\sum_{k=1}^{n}\left[\delta_{B_{k}(t)}(A)-\mathbf{P}\left\{B_{k}(t) \in A\right\}\right]\right) .
\end{aligned}
$$

Since $X_{n}(t, A)$ turns out to be extremely irregular as a measure in $A$ when $n$ gets large, one cannot expect the limit process $X(t)$ to be a measure-valued process. However, letting $\mathscr{S}$ be the Schwartz space, we can consider $X_{n}(t, \cdot)$ as a distribution-valued stochastic process $X_{n}(t)$ by setting

$$
\begin{aligned}
X_{n}(t)(\varphi) & =\int_{\mathbf{R}} \varphi(x) X_{n}(t, d x) \\
& =\frac{1}{\sqrt{n}}\left(\sum_{k=1}^{n}\left[\varphi\left(B_{k}(t)\right)-\mathbf{E} \varphi\left(B_{k}(t)\right)\right]\right), \quad \varphi \in \mathscr{S} .
\end{aligned}
$$

Both Itô [5] and Mitoma [7] show that there exists a distribution-valued stochastic process $X$ whose sample paths are elements in $C\left([0,1], \mathscr{S}^{\prime}\right)$ such that $X_{n}$ converges weakly to $X$.

One principal purpose of this paper is to extend the above result by assigning random mass to each individual. Let $B$ be a separable Banach space, $\left(X_{i}, i \geqslant 1\right)$ a sequence of independent identically distributed (i.i.d.) symmetric $B$-valued random variables and independent of all the Brownian motions. Define

$$
Z_{n}(t)=\frac{1}{\sqrt{n}} \sum_{i=1}^{n} X_{i} \delta_{B_{i}(t)}, \quad t \in[0,1] .
$$

Theorem 4.3 gives the limit law of the corresponding processes $Z_{n}, n \geqslant 1$.

For a fixed point $t_{0} \in[0,1]$, we have $Z_{n}\left(t_{0}\right)=\frac{1}{\sqrt{n}} \sum_{i=1}^{n} X_{i} \delta_{B_{i}\left(t_{0}\right)}, n \geqslant 1$. This is a sequence of normalized sums of vector random measures, and has a more general form. Let $\left(F_{i}, i \geqslant 1\right)$ be a sequence of i.i.d. and symmetric independently scattered (s.i.s.) $B$-valued random measures defined on $(\mathbf{R}, \mathscr{R})$. Let

$$
X_{n}=\frac{1}{\sqrt{n}} \sum_{i=1}^{n} F_{i}
$$


In Section 3 we shall be devoted to the study of the limit law for the $X_{n}$, and our basic ideas differ from D. H. Thang's [8] in that we regard $X_{n}$ as random linear functionals defined on the Schwartz space $\mathscr{S}$.

Section 2 contains some notation about vector random measures and Schwartz distribution spaces. Lemma 2.1, Proposition 2.1 and Proposition 4.1 are of fundamental importance for weak convergence of distributionvalued random variables; while Proposition 2.2 shows how to realize random linear functionals.

Throughout the paper, unless specifically stated otherwise, $c$ will denote a positive constant, which may be different from line to line.

2. Basic concepts and preliminary statements. Let $B$ be a separable Banach space and $(\mathbf{R}, \mathscr{R}, \mu)$ Lebesgue measure space. A set function $F: \mathscr{R} \rightarrow L_{0}(\Omega, \mathscr{F}, \mathbf{P} ; B)$ is called a $B$-valued s.i.s. random measure on $\mathscr{R}$ if

(i) for every sequence $\left(E_{n}\right)$ of disjoint sets from $\mathscr{R}$ the random variables $F\left(E_{1}\right), F\left(E_{2}\right), \ldots$ are symmetric and independent,

(ii) for every sequence $\left(E_{n}\right)$ of disjoint sets from $\mathscr{R}$ we have

$$
F\left(\bigcup_{n=1}^{\infty} E_{n}\right)=\sum_{n=1}^{\infty} F\left(E_{n}\right)
$$

a.s. in the norm topology of $B$.

The Lebesgue measure $\mu$ on $\mathscr{R}$ is called a control measure for $F$ if $F(E)=0$ a.s. whenever $\mu(E)=0$.

We can define random integrals of real-valued functions with respect to vector-valued s.i.s. random measures as usual. Suppose that $F$ is a $B$-valued s.i.s. random measure with the control measure $\mu$. If $f$ is a real-valued simple function defined on $\mathbf{R}, f=\sum_{i=1}^{n} a_{i} I_{A_{i}}$, where $A_{i}$ are disjoint sets, then the integral with respect to $F$ is defined by $\int_{\mathbf{R}} f d F=\sum_{i=1}^{n} a_{i} F\left(A_{i}\right)$. Moreover, a function $f$ on $\mathbf{R}$ is said to be $F$-integrable if there exists a sequence of simple functions $f_{n}$ such that

(i) $f_{n}(t) \rightarrow f(t), \mu$-a.e.,

(ii) the sequence $\int_{\mathbf{R}} f_{n} d F$ converges in probability.

If $f$ is $F$-integrable we put $\int_{\mathbf{R}} f d F=\mathbf{P}-\lim _{n \rightarrow \infty} \int_{\mathbf{R}} f_{n} d F$.

By saying that $\left\{F_{n}, n \geqslant 1\right\}$ are i.i.d. random measures we mean that $\left\{F_{n}(E), E \in \mathscr{R}\right\}, n \geqslant 1$, are i.i.d. as a sequence of random processes on $(\Omega, \mathscr{F}, \mathbb{P})$ indexed by $\sigma$-field $\mathscr{R}$. We refer to Theorem 4.3 in [8] for comparsion. There that $\left\{F_{n}, n \geqslant 1\right\}$ are independent copies of $F$ only means that for each $E\left\{F_{n}(E), n \geqslant 1\right\}$ are independent and have the same distributions as $F(E)$. Suppose that $\left\{F, F_{n}, n \geqslant 1\right\}$ is a sequence of $B$-valued s.i.s. random measures on $\mathscr{R}$. Note for any finite sequence $\left(E_{1}, E_{2}, \ldots, E_{k}\right)$ in $\mathscr{R}$ there exists a finite family $\mathscr{A}_{p}=\left(A_{1}, A_{2}, \ldots, A_{p}\right)$ of disjoint sets such that each $E_{i}$ is the union of some members from the family $\mathscr{A}_{p}$. This implies that each $F_{n}\left(E_{i}\right)$ can be expressed as a linear combination of $\left\{F_{n}\left(A_{i}\right), 1 \leqslant i \leqslant p\right\}$, 
$F_{n}\left(E_{i}\right)=\sum_{j=1}^{p} b_{i j} F_{n}\left(A_{j}\right)$, where $b_{i j}=0$ or 1 and do not depend on $n$. Consequently, for all $n \geqslant 1$

$$
\left(\begin{array}{c}
F_{n}\left(E_{1}\right) \\
\vdots \\
F_{n}\left(E_{k}\right)
\end{array}\right)=B\left(\begin{array}{c}
F_{n}\left(A_{1}\right) \\
\vdots \\
F_{n}\left(A_{p}\right)
\end{array}\right),
$$

where $B=\left(b_{i j}\right)$. From this we see that «for each $E \in \mathscr{R}, F_{n}(E)$ has the same distribution as $F(E) \gg$ is equivalent to saying that $\left\{F_{n}(E), E \in \mathscr{R}\right\}$ has the same distribution as $\{F(E), E \in \mathscr{R}\}$.

Now assume that we are given an s.i.s. random measure $F$ and a sequence $\left\{F_{n}, n \geqslant 1\right\}$ of independent copies of $F$. Define $X_{n}=\frac{1}{\sqrt{n}} \sum_{i=1}^{n} F_{i}$, $n \geqslant 1$. As usual we hope to regard $F$ as a random vector in some topological vector space. Denoting by $\mathfrak{F}(\mathscr{R} ; B)$ the set of all countably additive set functions from $\mathscr{R}$ into $B$, at a glance such a topological vector space should be $\mathfrak{F}(\mathscr{R} ; B)$ equipped with the weak topology (i.e., $F_{n}$ converges weakly to $F$ if for each bounded continuous function $f, \lim _{n \rightarrow \infty} \int_{\mathbf{R}} f d F_{n}=\int_{\mathbf{R}} d F$ in the norm of $B$, see [1] for the weak topology). Unfortunately, the preceding definition of random measure does not guarantee $F(\omega) \in \mathfrak{F}(\mathscr{R} ; B)$ for all or almost all $\omega$ in $\Omega$. In addition, it is very hard for us to discuss the weak topology in $\mathfrak{F}(\mathscr{R} ; B)$ itself. To avoid the difficulties we shall turn to the study of a sequence of random linear mappings induced by $X_{n}$ as Itô [5] and Mitoma [7] did.

Next we recall some known facts on the Schwartz distributions. $\mathscr{D}$ and $\mathscr{D}^{\prime}$ denote the $C^{\infty}$-functions of compact support and the Schwartz distributions respectively. $H_{n}$ is the Hermite polynomial of degree $n$ and $h_{n}(x)$ is the corresponding Hermite functions, i.e., $h_{n}(x)=c_{n} H_{n}(x) e^{-x^{2} / 2}$, $n=0,1,2, \ldots$, where $c_{n}=\left(\frac{\pi}{2} 2^{n} n !\right)^{-1 / 2}$. The Hermite functions form an orthonormal basis (ONB) in $L_{2}(\mathbf{R})$. The $p$-norm $\|\cdot\|_{p}$ in $L_{2}(\mathbf{R})$ and the $(-p)$-norm $\|\cdot\|_{-p}$ in $\mathscr{D}^{\prime}$ are defined as follows:

$$
\begin{aligned}
\|\varphi\|_{p}^{2} & =\sum_{n=0}^{\infty}\left(\varphi, h_{n}\right)^{2}(2 n+1)^{p}, \\
\|f\|_{-p}^{2} & =\sum_{n=0}^{\infty} h_{n}^{2}(2 n+1)^{-p},
\end{aligned}
$$

where $p=0,1,2, \ldots$ and $f(\varphi)$ denotes the value evaluated at $\varphi \in \mathscr{D}$.

It is clear that

$$
\|f\|_{p}=\sup \left\{|f(\varphi)|: \varphi \in \mathscr{D}, \quad\|\varphi\|_{p} \leqslant 1\right\},
$$

which implies $|f(\varphi)| \leqslant\|f\|_{-p}\|\varphi\|_{p}, f \in \mathscr{D}^{\prime}, \varphi \in \mathscr{D}$. Define $\mathscr{S}_{p},(\varphi, \psi)_{p}, \mathscr{S}_{p}^{\prime}$ and $(f, g)_{-p}$ as follows:

$$
\begin{aligned}
& \mathscr{S}_{p}=\left\{\varphi \in L_{2}(\mathbf{R}):\|\varphi\|_{p}<\infty\right\}, \\
& \mathscr{S}_{p}^{\prime}=\left\{f \in \mathscr{D}^{\prime}:\|f\|_{-p}<\infty\right\},
\end{aligned}
$$




$$
\begin{aligned}
(\varphi, \psi)_{p} & =\sum_{n=0}^{\infty}\left(\varphi, h_{n}\right)\left(\psi, h_{n}\right)(2 n+1)^{p} \\
(f, g)_{-p} & =\sum_{n=0}^{\infty} f\left(h_{n}\right) g\left(h_{n}\right)(2 n+1)^{-p} .
\end{aligned}
$$

Then $\left(\mathscr{S}_{p},(\cdot, \cdot)_{p}\right)$ and $\left(\mathscr{S}_{p}^{\prime},(\cdot, \cdot)_{-p}\right)$ are Hilbert spaces with the norms $\|\cdot\|_{p}$ and $\|\cdot\|_{-p}$ respectively. As $p$ increases, $\|\cdot\|_{p}$ increases and $\|\cdot\|_{-p}$ decreases. So $\mathscr{S}_{p}$ decreases and $\mathscr{S}_{p}^{\prime}$ increases. The intersection $\bigcap_{p} \mathscr{S}_{p}$ coincides with the space $\mathscr{S}$ of rapidly decreasing functions and the union $\bigcup_{p} \mathscr{S}_{p}^{\prime}$ with the space $\mathscr{S}^{\prime}$ of tempered distributions. It follows from the definition that $\mathscr{S}_{0}=L_{2}(\mathbf{R}),\|\cdot\|_{0}=\|\cdot\|$ and $(\cdot, \cdot)_{0}=(\cdot, \cdot)$. By identifying $\psi \in L_{2}(\mathbf{R})$ with $[\psi] \in \mathscr{D}^{\prime}$, where $[\psi](\varphi)=:(\psi, \varphi)$, we have $L_{2}=\mathscr{S}_{0}^{\prime},\|\cdot\|=\|\cdot\|_{-0}$ and $(\cdot, \cdot)=(\cdot, \cdot)_{-0}$. Hence

$\mathscr{D} \subset \mathscr{S} \subset \cdots \subset \mathscr{S}_{2} \subset \mathscr{S}_{1} \subset \mathscr{S}_{0}=L_{2}(\mathbf{R})=\mathscr{S}_{0}^{\prime} \subset \mathscr{S}_{1}^{\prime} \subset \cdots \subset \mathscr{S}^{\prime} \subset \mathscr{D}^{\prime}$.

In other words, $\mathscr{S}$ and $\mathscr{S}^{\prime}$ are nuclear Frechet spaces.

Let $\mathscr{L}(\mathscr{S}, B)$ be the set of all continuous linear mappings from $\mathscr{S}$ into $B$. Let us equip $\mathscr{L}(\mathscr{S}, B)$ with the strong topology, i.e., the topology of uniform convergence on all bounded subsets of $\mathscr{S}$, and denote it by $\mathscr{L}_{b}(\mathscr{S}, B)$. This is a completely regular topological vector space. Analogously, $\mathscr{L}_{b}\left(\mathscr{S}_{p}, B\right)$ is a Banach space.

The following lemma due to Le Cam (see [10, Theorem 6.7]) is an extension of Prokhorov's theorem.

Lemma 2.1. Let $E$ be a completely regular topological space such that all compact sets are metricable. If $\left\{P_{n}, n \geqslant 1\right\}$ is a sequence of probability measures on $E$ which is uniformly tight, then there exist a subsequence $\left(n_{k}\right)$ and a probability measure $Q$ such that $P_{n_{k}} \Rightarrow Q$.

To apply Lemma 2.1 to $\mathscr{L}_{b}(\mathscr{S}, B)$ we need

Proposition 2.1. If $K$ is compact in $\mathscr{L}_{b}(\mathscr{S}, B)$, then there exists a natural number $p$ such that $K$ is compact in $\mathscr{L}_{b}\left(\mathscr{S}_{p}, B\right)$.

$\mathrm{P}$ r o of. For each $\varphi \in \mathscr{S}$ define $\pi_{\varphi}: \mathscr{L}_{b}(\mathscr{S}, B) \rightarrow B$ as $\pi_{\varphi} T=T \varphi$, $T \in \mathscr{L}_{b}(\mathscr{S}, B)$.

If $T_{n} \rightarrow T$ in the strong topology, then $\left\|\pi_{\varphi} T_{n}-\pi_{\varphi} T\right\|=\left\|T_{n} \varphi-T \varphi\right\| \rightarrow 0$. So $\pi_{\varphi}$ is a continuous linear mapping. Thus $\left\{\pi_{\varphi} T, T \in K\right\}$ is compact in $B$ whenever $K$ is compact in $\mathscr{L}_{b}(\mathscr{S}, B)$. This implies

$$
\sup _{\varphi \in \mathscr{S}} \sup _{T \in K}\|T \varphi\|<\infty .
$$

By the Banach-Steinhaus theorem (see [9, Theorem 33.1]), $K$ is equicontinuous in $\mathscr{L}_{b}(\mathscr{S}, B)$, i.e., for any neighborhood of zero $V$ in $B$ there is a neighborhood of zero $U$ in $\mathscr{S}$ such that for all mappings $T \in K$ we have: $\varphi \in U$ implies $T \varphi \in V$. In particular, letting $V=\{x:\|x\| \leqslant 1\}, U$ contains 
a basis element, say $\left\{\varphi:\|\varphi\|_{q}<\varepsilon\right\}$, such that $T \varphi \in V$. Thus

$$
\sup _{T \in K}\|T \varphi\| \leqslant \frac{c\|\varphi\|_{q}}{\varepsilon} \text { for all } \varphi \in \mathscr{S} \text {. }
$$

For such a $q$ there exists a natural number $p>q$ such that the natural embedding $i: \mathscr{S}_{p} \rightarrow \mathscr{S}_{q}$ is nuclear, and hence compact. The closure $S$ of $\left\{\varphi:\|\varphi\|_{p} \leqslant 1\right\}$ is compact in $\mathscr{S}_{q}$ and so is $\{T \varphi, T \in K, \varphi \in S\}$.

On the other hand, $\sup _{T \in K}\|T \varphi-T \psi\| \leqslant c\|\varphi-\psi\|_{q}$. If we consider $K$ as a subset of $C(S, B)$, the set of continuous mappings from $S$ into $B$, the required result is easily obtained.

Let us turn back to the existence of the random integral $\int_{\mathbf{R}} \varphi d F$ for any given $\varphi \in \mathscr{S}$ and s.i.s. random measure $F$ on $(\mathbf{R}, \mathscr{R})$. Suppose that $B$ is of type 2 space and $\mathbf{E}\|F(E)\|^{2} \leqslant c \mu(E)$ for all $E \in \mathscr{R}$, then for any simple function $\varphi_{n}(t)=\sum_{i=1}^{n} a_{i} I_{E_{i}}(t)$,

$$
\begin{aligned}
\mathbf{E}\left\|\int_{\mathbf{R}} \varphi_{n}(t) d F\right\|^{2} & =\mathbf{E}\left\|\sum_{i=1}^{n} a_{i} F\left(E_{i}\right)\right\|^{2} \leqslant c \sum_{i=1}^{n} a_{i}^{2} \mathbf{E}\left\|F\left(E_{i}\right)\right\|^{2} \\
& \leqslant c \sum_{i=1}^{n} a_{i}^{2} \mu\left(E_{i}\right)=c \int_{\mathbf{R}} \varphi_{n}^{2}(t) d \mu(t) .
\end{aligned}
$$

Thus $\varphi$ must be integrable with respect to random measure $F$ and

$$
\mathbf{E}\left\|\int_{\mathbf{R}} \varphi(t) d F\right\|^{2} \leqslant c \int_{\mathbf{R}} \varphi^{2}(t) d \mu(t)=c\|\varphi\|_{0}^{2} .
$$

Whenever the random integral $\int_{\mathbf{R}} \varphi d F$ exists for each $\varphi \in \mathscr{S}$ can we define a random linear mapping $T_{F}: T_{F}(\varphi)=\int_{\mathbf{R}} \varphi d F$ from $\mathscr{S}$ into $B$.

Proposition 2.2. Let $Y=\{\dot{Y}(\varphi): \varphi \in \mathscr{S}\}$ be a family of $B$-valued random variables such that $Y(\varphi)$ is

(L) almost linear,

$$
Y\left(C_{1} \varphi_{1}+C_{2} \varphi_{2}\right)=C_{1} Y\left(\varphi_{1}\right)+C_{2} Y\left(\varphi_{2}\right) \text { a.s. }
$$

where the exceptional $\omega$-set may depend on the choice of $\left(C_{1}, \varphi_{1} ; C_{2}, \varphi_{2}\right)$;

(B) p-bounded,

$$
\mathbf{E}\|Y(\varphi)\|^{2} \leqslant\|\varphi\|_{p}^{2}
$$

Then $Y$ has an $\mathscr{L}_{b}\left(\mathscr{S}_{p+2}, B\right)$ regularization $X$ satisfying

$$
\mathbf{E}\|X\|_{-p-2}^{2} \leqslant \frac{\pi^{2} c}{8}
$$

Here and in the sequel we denote by $\|X\|_{-p-2}$ the operator norm of $X$. in $\mathscr{L}_{b}\left(\mathscr{S}_{p+2}, B\right)$.

P r o of. The conditions (L) and (B) imply that the map $Y: \varphi \rightarrow$ $Y(\varphi)$ is a bounded linear operator from the pre-Hilbert space $\left(\mathscr{S},\|\cdot\|_{p}\right)$ into $L_{2}(\Omega, \mathscr{F}, \mathbf{P} ; B)$. Since $\mathscr{S}$ is dense in $\mathscr{S}_{p}$, this map can be extended to a unique linear operator from $\mathscr{S}_{p}$ into $\mathrm{L}_{2}(\Omega, \mathscr{F}, \mathbf{P} ; B)$ still denoted by the same 
notation $Y$. Let $h_{n}$ be the Hermite functions, then $\varepsilon_{n}=(2 n+1)^{(p+2) / 2} h_{n} \in$ $\mathscr{S}_{p}, n=0,1,2, \ldots$, form an ONB in $\mathscr{S}_{p+2}$. The ONB in $\mathscr{S}_{p+2}^{\prime}$ dual to $\varepsilon_{n}$ is denoted by $\left\{e_{n}\right\}$. It is clear that

$$
\mathbf{E} \sum_{n=1}^{\infty}\left\|Y\left(\varepsilon_{n}\right)\right\|^{2} \leqslant c \sum_{n=1}^{\infty}\left\|\varepsilon_{n}\right\|_{p}^{2}<\infty .
$$

This implies that $\mathbf{P}\left(\Omega_{1}\right)=1$, where $\Omega_{1}=\left\{\omega \in \Omega: \sum_{n=1}^{\infty}\left\|Y\left(\varepsilon_{n}\right)(\omega)\right\|^{2}<\infty\right\}$. Define

$$
X(\omega)= \begin{cases}\sum_{n=1}^{\infty} Y\left(\varepsilon_{n}\right)(\omega) e_{n}, & \text { on } \Omega_{1}, \\ 0, & \text { otherwise. }\end{cases}
$$

Then $X(\omega) \in \mathscr{L}_{b}\left(\mathscr{S}_{p+2}, B\right)$ for every $\omega$ and $X(\omega)$ is measurable in $\omega$. Hence $X$ is an $\mathscr{L}_{b}\left(\mathscr{S}_{p+2}, B\right)$-valued variable and

$$
\begin{aligned}
\mathbf{E}\|X\|_{-p-2}^{2} & =\mathbf{E} \sup _{\|\varphi\|_{p+2} \leqslant 1}\|X(\omega)(\varphi)\|_{B}^{2} \\
& =\mathbf{E} \sup _{f} \sup _{\|\varphi\|_{p+2} \leqslant 1}\left|\sum_{n=1}^{\infty} f(Y(\varepsilon))(\omega)\left(e_{n}, \varphi\right)\right| \\
& \leqslant \sum_{n=1}^{\infty} \mathbf{E}\left\|X_{n}\right\|_{B}^{2} \leqslant \frac{\pi^{2} c}{8}
\end{aligned}
$$

Since $e_{m}\left(\varepsilon_{n}\right)=\delta_{m n}$ and $\mathbf{P}\left(\Omega_{1}\right)=1$, then $X(\varphi)=Y(\varphi)$ a.s. for $\varphi=\varepsilon_{n}$, $n=1,2, \ldots$, and so for every finite linear combination of $\left\{\varepsilon_{n}\right\}$ by $(\mathrm{L})$. For a general $\varphi \in \mathscr{S}_{p+2} \subset \mathscr{S}_{p}$ we have

$$
\left\|\varphi-\varphi_{n}\right\|_{p+2} \rightarrow 0 \quad(n \rightarrow \infty), \quad \varphi_{n}=: \sum_{k=0}^{n}\left(\varphi, \varepsilon_{k}\right)_{p+2} \varepsilon_{k} .
$$

Since $X \in \mathscr{L}_{b}\left(\mathscr{S}_{p+2}, B\right)$, then $\left\|X(\varphi)-X\left(\varphi_{n}\right)\right\|=\|X\|_{-p-2} \| \varphi-$ $\varphi_{n} \|_{p+2} \rightarrow 0$ for every $\omega$. Also, we can use (B) to check that

$$
\mathbf{E}\left\|Y(\varphi)-Y\left(\varphi_{n}\right)\right\|^{2} \leqslant c\left\|\varphi-\varphi_{n}\right\|_{p}^{2} \leqslant c\left\|\varphi-\varphi_{n}\right\|_{p+2}^{2} \rightarrow 0 .
$$

Thus $X(\varphi)=Y(\varphi)$ a.s. for every $\varphi \in \mathscr{S}_{p+2}$.

3. The CLT for vector random measures. After having the preceding preparations, we can consider $X_{n}=\frac{1}{\sqrt{n}} \sum_{i=1}^{n} F_{i}$ as an $\mathscr{L}_{b}(\mathscr{S}, B)$ valued random vector by setting $X_{n}(\varphi)=\frac{1}{\sqrt{n}} \sum_{i=1}^{n} \int_{\mathbf{R}} \varphi d F_{i}, \varphi \in \mathscr{S}$. It is on this viewpoint that we base our studies of weak convergence for random measures.

Now we shall state and prove our main results at this area.

Theorem 3.1. Suppose that $B$ is the space of type $2,\left\{F, F_{n}, n \geqslant 1\right\}$, is a sequence of i.i.d. and s.i.s. random measures on $(\mathbf{R}, \mathscr{R}, \mu)$. If $\mathbf{E}\|F(E)\|^{4} \leqslant$ $c \mu^{2}(E)$, and $\left\{F(E) / \mu^{1 / 2}(E), E \in \mathscr{R}, \mu(E)>0\right\}$ is uniformly tight in $B$, then there exists an $\mathscr{L}_{b}(\mathscr{S}, B)$-valued Gaussian vector $G$ such that $\left\{X_{n}(\varphi), \varphi \in \mathscr{S}\right\}$ converges weakly to $G$. 
P r o o f. By Lemma 2.1 it suffices to verify the following two statements:

(i) $X_{n}$ is uniformly tight in $\mathscr{L}_{b}(\mathscr{S}, B)$, i.e., for any $\varepsilon>0$ there is a compact set $K \subset \mathscr{L}_{b}(\mathscr{S}, B)$ such that

$$
\sup _{n} \mathbf{P}\left\{\omega: X_{n}(\omega) \notin K\right\}<\varepsilon ;
$$

(ii) for each $f \in \mathscr{L}_{b}^{\prime}(\mathscr{S}, B), f\left(T_{F}\right)$ satisfies the classical central limit theorem.

Let us first prove (i). If $K$ is compact in $\mathscr{L}_{b}\left(\mathscr{S}_{q}, B\right)$ for some $q$, then $K$ is also compact in $\mathscr{L}_{b}(\mathscr{S}, B)$ since the injection of $\mathscr{L}_{b}\left(\mathscr{S}_{q}, B\right)$ into $\mathscr{L}_{b}(\mathscr{S}, B)$ is continuous.

In the scalar case (i.e., $B=\mathbf{R}$ ) for every $p \in \mathbf{N}$ there is a $q>p, q \in \mathbf{N}$, such that the natural embedding $i: \mathscr{S}_{q} \rightarrow \mathscr{S}_{p}$ is nuclear and so compact, thus its adjoint $i^{*}: \mathscr{S}_{p}^{\prime} \rightarrow \mathscr{S}_{q}^{\prime}$ is also compact (see [2, Theorem VI.5.2]). Equivalently, any bounded subset of $\mathscr{S}_{p}^{\prime}$ is relatively compact in $\mathscr{S}_{q}^{\prime}$.

In the general case (i.e., $B$ a separable Banach space), however, it is possible that a bounded subset of $\mathscr{L}_{b}\left(\mathscr{S}_{p}, B\right)$ is not necessarily relatively compact in $\mathscr{L}_{b}\left(\mathscr{S}_{q}, B\right)$. So we need to make some necessary changes as follows.

For each $p \in \mathbf{N}, k>0$ and compact subset $K_{B}$ in $B$, define

$$
K=\left\{T:\|T\|_{-p} \leqslant k\right\} \cap\left\{T:\left\{T \varphi,\|\varphi\|_{q} \leqslant 1\right\} \subset K_{B}\right\},
$$

where $q$ is such that $i: \mathscr{S}_{q} \rightarrow \mathscr{S}_{p}$ is nuclear.

We claim that $K$ is also relatively compact in $\mathscr{L}_{b}\left(\mathscr{S}_{q}, B\right)$.

Indeed, let $S$ be the closure of $\left\{\varphi:\|\varphi\|_{q} \leqslant 1\right\}$ in $\mathscr{S}_{p}$, then $S$ is compact in $\mathscr{S}_{p}$. Since $\|T \varphi-T \psi\| \leqslant k\|\varphi-\psi\|_{q}$, the set $K$ is equicontinuous in $\mathscr{L}_{b}\left(\mathscr{S}_{q}, B\right)$. In addition, $\{T \varphi: T \in K ; \varphi \in S\} \subset K_{B}$. Thus considering $K$ as a subset of $C(S, B)$, the set of continuous mappings from $S$ into $B$, we easily deduce that $K$ is relatively compact in $C(S, B)$, so in $\mathscr{L}_{b}\left(\mathscr{S}_{q}, B\right)$.

Thus in order to verify (i) it is enough to show

(i') for each $\varepsilon>0$, there exist $p, q \in \mathbf{N}(q>p), k>0$ and a compact subset $K_{B} \subset B$ such that

$$
\begin{aligned}
& \sup _{n} \mathbf{P}\left\{\omega:\left\|X_{n}\right\|_{-p}>k\right\}<\varepsilon, \\
& \sup _{n} \mathbf{P}\left\{\omega: X_{n}(\varphi) \notin K_{B}, \text { for some } \varphi,\|\varphi\|_{q} \leqslant 1\right\}<\varepsilon .
\end{aligned}
$$

Equivalently, according to a well-known fact (see [6, Lemma 2.2]): a subset $K_{B}$ of $B$ is relatively compact if and only if it is bounded and for each $\varepsilon>0$ there is a finite dimensional closed subspace $F$ of $B$ such that $\sup _{x \in K_{B}} q_{F}(x)<\varepsilon$, where $q_{F}$ is the quotient norm, we need only to check the following statement. 
For each $\varepsilon>0$ there exist $p, q \in \mathbf{N}(q>p), k>0$ and a finite dimensional closed subspace $F$ of $B$ such that

$$
\begin{aligned}
& \sup _{n} \mathbf{P}\left\{\omega:\left\|X_{n}\right\|_{-p}>k\right\}<\varepsilon, \\
& \sup _{n} \mathbf{P}\left\{\omega: \sup _{\|\varphi\|_{q} \leqslant 1} q_{F}\left(X_{n}(\varphi)\right)>\varepsilon\right\}<\varepsilon .
\end{aligned}
$$

This will in turn be done in two steps.

$\mathrm{S} \mathrm{t}$ e p 1: for each $\varepsilon>0$ there exist $p \in \mathbf{N}$ and $k>0$ such that

$$
\sup _{n} \mathbf{P}\left\{\omega: \sup _{\|\varphi\|_{p} \leqslant 1}\left\|X_{n}(\varphi)\right\|>k\right\}<\varepsilon .
$$

The idea behind the proof of (3.7) is similar to that of Theorem 6.12 in [10].

1 ) For any $\varepsilon>0$, there exist $m$ and $\delta>0$ such that

$$
\|\varphi\|_{m}<\delta \quad \text { implies } \sup _{n} \mathbf{E}\left(\left\|X_{n}(\varphi)\right\| \wedge 1\right)<\varepsilon .
$$

To see this, consider the function $F(\varphi)=\sup _{n} \mathbf{E}\left(\left\|X_{n}(\varphi)\right\| \wedge 1\right), \varphi \in \mathscr{S}$. Then

(i) $F(0)=0$,

(ii) $F(\varphi) \geqslant 0$ and $F(\varphi)=F(-\varphi)$,

(iii) $F(a \varphi)<F(b \varphi)$ if $|a|<|b|$,

(iv) $F$ is lower-semicontinuous on $\mathscr{S}$.

Indeed, if $\varphi_{j} \rightarrow \varphi$ in $\mathscr{S}$, then $X_{n}\left(\varphi_{j}\right) \rightarrow X_{n}(\varphi)$ in $\mathrm{七}_{0}(\Omega, \mathscr{F}, \mathbf{P} ; B)$, and hence $\left\|X_{n}\left(\varphi_{j}\right)\right\| \wedge 1 \rightarrow\left\|X_{n}(\varphi)\right\| \wedge 1$ in probability. Then

$$
\begin{aligned}
F(\varphi) & =\sup _{n} \mathbf{E}\left(\left\|X_{n}(\varphi)\right\| \wedge 1\right) \leqslant \sup _{n} \liminf _{j} \mathbf{E}\left(\left\|X_{n}\left(\varphi_{j}\right)\right\| \wedge 1\right) \\
& \leqslant \liminf _{j} \sup _{n} \mathbf{E}\left(\left\|X_{n}\left(\varphi_{j}\right)\right\| \wedge 1\right)=\liminf _{j} F\left(\varphi_{j}\right) .
\end{aligned}
$$

(v) $\lim _{n} F(\varphi / n)=0$.

For this note that for any given $\varepsilon>0$ and $\varphi \in \mathscr{S}$ there exists a $k>0$ such that

$$
\sup _{n} \mathbf{P}\left\{\omega:\left\|X_{n}(\varphi)\right\|>k\right\} \leqslant \frac{\mathbf{E}\left\|X_{n}(\varphi)\right\|^{2}}{k^{2}}=\frac{\mathbf{E}\left\|\int_{\mathbf{R}} \varphi d F\right\|^{2}}{k^{2}} \leqslant \frac{c\|\varphi\|_{0}^{2}}{k^{2}}<\frac{\varepsilon}{2} .
$$

Choose $M$ large enough so that $k / M<\varepsilon / 2<1$, then

$$
F\left(\frac{\varphi}{M}\right)=\sup _{n} \mathbf{E}\left(\left\|X_{n}\left(\frac{\varphi}{M}\right)\right\| \wedge 1\right) \leqslant \sup _{n} \mathbf{P}\left\{\omega: \frac{\left\|X_{n}(\varphi)\right\|}{M}>\frac{k}{M}\right\}+\frac{k}{M}<\varepsilon .
$$

Let $V=\{\varphi: F(\varphi) \leqslant \varepsilon\}, V$ is closed symmetric absorbing. We claim that it is a neighborhood of 0 . Indeed $\mathscr{S}=\cup_{n} n V$, so by the Baire category theorem, one and hence all, of the $n V$ must have a non-empty interior. In particular, $\frac{1}{2} V$ does. Then $V \subset \frac{1}{2} V-\frac{1}{2} V$ must contain a neighborhood of 0 . This in turn must contain an element of basis, say $\left\{\|\varphi\|_{m}<\delta\right\}$. This proves 1$)$. 
2) For all $n \geqslant 1$

$$
\mathbf{E} \sup _{f \in B^{\prime}} \operatorname{Re}\left(1-e^{i\left\langle f, X_{n}(\varphi)\right\rangle}\right) \leqslant 2 \varepsilon\left(1+\frac{\|\varphi\|_{m}^{2}}{\delta^{2}}\right), \quad \varphi \in \mathscr{S} .
$$

In fact, in view of 1 ) we have

$$
\operatorname{Re}\left(1-e^{i\left\langle f, X_{n}(\varphi)\right\rangle}\right)=1-\cos \left\langle f, X_{n}(\varphi)\right\rangle \leqslant \frac{1}{2}\left(\left\langle f, X_{n}(\varphi)\right\rangle^{2} \wedge 2\right) .
$$

If $\|\varphi\|_{m}<\delta$, then

$$
\begin{aligned}
\mathbf{E} \sup _{f \in B^{\prime}} \operatorname{Re}\left(1-e^{i\left\langle f, X_{n}(\varphi)\right\rangle}\right) & \leqslant \frac{1}{2} \mathbf{E} \sup _{f \in B^{\prime}}\left(\left\langle f, X_{n}(\varphi)\right\rangle^{2} \wedge 2\right) \\
& \leqslant 2 \mathbf{E} \sup _{f \in B^{\prime}}\left(\left|\left\langle f, X_{n}(\varphi)\right\rangle\right| \wedge 1\right)<2 \varepsilon .
\end{aligned}
$$

On the other hand, if $\|\varphi\|_{m} \geqslant \delta$, replace $\varphi$ by $\psi=\delta \varphi /\|\varphi\|_{m}$,

$\mathbf{E} \sup _{f \in B^{\prime}} \operatorname{Re}\left(1-e^{i\left\langle f, X_{n}(\varphi)\right\rangle}\right) \leqslant \frac{1}{2} \mathbf{E} \sup _{f \in B^{\prime}}\left\langle f, X_{n}(\varphi)\right\rangle^{2} \frac{\|\varphi\|_{m}^{2}}{\delta^{2}} \wedge 2 \leqslant 2 \varepsilon \frac{\|\varphi\|_{m}^{2}}{\delta^{2}}$.

The required (3.9) is proved.

3) There are $p>m$ and $M$ such that for all $n$ and $k>0$,

$$
\mathbf{E}\left(1-e^{-k^{-1} \sup _{\|\varphi\|_{p} \leqslant 1}\left\|X_{n}(\varphi)\right\|^{2}}\right) \leqslant 2 \varepsilon\left(1+\frac{M}{k \delta^{2}}\right) .
$$

Indeed, there exists a $p>m$ such that the natural embedding $i: \mathscr{S}_{p} \rightarrow \mathscr{S}_{m}$ is a Hilbert-Schmidt operator. Let $\left(e_{j}\right)$ be a complete orthogonal normed system (CONS) in $\mathscr{S}$ relative to $\|\cdot\|_{p}$. Let $Y_{1}, Y_{2}, \ldots$ be i.i.d. $N(0,2 / k)$ random variables, and put $\Phi_{N}=\sum_{j=1}^{N} Y_{j} e_{j}$. Then by 2$)$

$$
\begin{aligned}
\mathbf{E} \sup _{f \in B^{\prime}} \operatorname{Re}\left(1-e^{i\left\langle f, X_{n}\left(\Phi_{N}\right)\right\rangle}\right) & =\mathbf{E}_{Y} \mathbf{E}_{X}\left(\sup _{f \in B^{\prime}} \operatorname{Re}\left(1-e^{i\left\langle f, X_{n}\left(\Phi_{N}\right)\right\rangle}\right) \mid Y\right) \\
& \leqslant \mathbf{E} 2 \varepsilon\left(1+\frac{\left\|\Phi_{N}\right\|_{m}^{2}}{\delta^{2}}\right) .
\end{aligned}
$$

On the other hand,

$$
\begin{aligned}
\mathbf{E} \sup _{f \in B^{\prime}} \operatorname{Re}\left(1-e^{i\left\langle f, X_{n}\left(\Phi_{N}\right)\right\rangle}\right) & =\mathbf{E} \sup _{f \in B^{\prime}} \operatorname{Re}\left(1-e^{i \sum_{j=1}^{N}\left\langle f, X_{n}\left(e_{j}\right)\right\rangle Y_{j}}\right) \\
& \geqslant \mathbf{E} \sup _{f \in B^{\prime}} \mathbf{E}\left(\operatorname{Re}\left(1-e^{i \sum_{j=1}^{N}\left\langle f, X_{n}\left(e_{j}\right)\right\rangle Y_{j}}\right) \mid X\right) .
\end{aligned}
$$

But, given $X$, the conditional distribution of $\sum_{j=1}^{N}\left\langle f, X_{n}\left(e_{j}\right)\right\rangle Y_{j}$ is $N\left(0,(2 / k) \sum_{j=1}^{N}\left\langle f, X_{n}\left(e_{j}\right)\right\rangle^{2}\right)$. So the right-hand side of the last inequality is

$$
\mathbf{E} \sup _{f \in B^{\prime}}\left(1-e^{-k^{-1} \sum_{j=1}^{N}\left\langle f, X_{n}\left(e_{j}\right)\right\rangle^{2}}\right) \text {. }
$$


Since

$$
\begin{aligned}
\sup _{\|\varphi\|_{p} \leqslant 1}\left\|X_{n}(\varphi)\right\| & =\sup _{\|\varphi\|_{p} \leqslant 1} \sup _{f \in B^{\prime}}\left\langle f, X_{n}(\varphi)\right\rangle \\
& =\sup _{f \in B^{\prime}} \sup \left\{\sum_{i, j=1}^{\infty} a_{i}\left\langle f, X_{n}\left(e_{j}\right)\right\rangle, \sum_{i=1}^{\infty} a_{i}^{2} \leqslant 1\right\} \\
& =\sup _{f \in B^{\prime}}\left(\sum_{j=1}^{\infty}\left\langle f, X_{n}\left(e_{j}\right)\right\rangle^{2}\right)^{1 / 2},
\end{aligned}
$$

then setting $M=: 2 \sum_{j=1}^{\infty}\left\|e_{j}\right\|_{m}^{2}$, we easily obtain (3.10) as follows:

$$
\begin{aligned}
& \mathbf{E}\left(1-e^{-k^{-1} \sup _{\|\varphi\|_{p} \leqslant 1}\left\|X_{n}(\varphi)\right\|^{2}}\right)=\mathbf{E}\left(1-e^{-k^{-1} \sup _{\|\varphi\|_{p} \leqslant 1}\left\|X_{n}(\varphi)\right\|^{2}}\right) \\
& =\mathbf{E}\left(1-e^{-k^{-1} \sup _{f \in B^{\prime}} \sum_{j=1}^{\infty}\left\langle f, X_{n}\left(e_{j}\right)\right\rangle^{2}}\right) \\
& =\mathbf{E} \sup _{f \in B^{\prime}}\left(1-e^{-k^{-1} \sum_{j=1}^{\infty}\left\langle f, X_{n}\left(e_{j}\right)\right\rangle^{2}}\right) \\
& =\mathbf{E} \sup _{f \in B^{\prime}} \lim _{N \rightarrow \infty}\left(1-e^{-k^{-1} \sum_{j=1}^{N}\left\langle f, X_{n}\left(e_{j}\right)\right\rangle^{2}}\right) \\
& \leqslant \lim _{N \rightarrow \infty} \mathbf{E} \sup _{f \in B^{\prime}}\left(1-e^{-k^{-1} \sum_{j=1}^{N}\left\langle f, X_{n}\left(e_{j}\right)\right\rangle^{2}}\right) \leqslant 2 \varepsilon\left(1+\frac{M}{k \delta^{2}}\right) .
\end{aligned}
$$

4) Markov's inequality gives

$$
\mathbf{P}\left\{\omega: \sup _{\|\varphi\|_{p} \leqslant 1}\left\|X_{n}(\varphi)\right\|^{2}>k\right\} \leqslant \frac{e}{e-1} \mathbf{E}\left(1-e^{-k^{-1} \sup _{\|\varphi\|_{p} \leqslant 1}\left\|X_{n}(\varphi)\right\|^{2}}\right) .
$$

The proof of (3.7) is complete.

$\mathrm{S} t$ e $\mathrm{p}$ 2: for any $\varepsilon>0$ there exists a finite dimensional closed subspace $F$ in $B$ such that

$$
\sup _{n} \mathbf{P}\left\{\omega: \sup _{\|\varphi\|_{q} \leqslant 1} q_{F}\left(X_{n}(\varphi)\right)>\varepsilon\right\}<\varepsilon
$$

where $q$ is determined by $p$ of Step 1 .

The proof for (3.12) involves the same idea as for (3.7), but requires stronger assumptions. Given $\varepsilon>0$, let $p$ be chosen in Step 1. There exists $q>p$ such that if $\left(e_{j}\right)$ is a CONS in $\mathscr{S}_{q} \subset \mathscr{S}_{p}$, then $C^{2}=: \sum_{j=1}^{\infty}\left\|e_{j}\right\|_{p}^{2}<\infty$. Letting $Y_{1}, Y_{2}, \ldots$ be a sequence of i.i.d. normal random variables $N(0,2 / \varepsilon)$, we have

$$
\begin{aligned}
\mathbf{P}\left\{\omega:\left\|\sum_{j=1}^{\infty} Y_{j} e_{j}\right\|_{p}>\frac{4 C}{\varepsilon}\right\} & \leqslant\left(\frac{\varepsilon}{4 C}\right)^{2} \mathbf{E}\left\|\sum_{j=1}^{\infty} Y_{j} e_{j}\right\|_{p}^{2} \\
& \leqslant\left(\frac{\varepsilon}{4 C}\right)^{2} \mathbf{E} \sum_{j=1}^{\infty} Y_{j}^{2}\left\|e_{j}\right\|_{p}^{2} \leqslant \frac{\varepsilon}{8} .
\end{aligned}
$$


We continue to prove that there exists a finite dimensional closed subspace $F$ in $B$ such that

$$
\sup _{\|\varphi\|_{p} \leqslant 4 C / \varepsilon} \sup _{n} \mathbf{P}\left\{\omega: q_{F}\left(X_{n}(\varphi)\right)>\frac{\varepsilon}{8}\right\}<\frac{\varepsilon}{8}
$$

Since $B$ is the space of type 2 , then for any closed subspace $F$ in $B$, $B / F$ is also of type 2 . So

$$
\mathbf{P}\left\{\omega: q_{F}\left(X_{n}(\varphi)\right)>\frac{\varepsilon}{8}\right\} \leqslant \frac{64}{\varepsilon^{2}} \mathbf{E} q_{F}^{2}\left(X_{n}(\varphi)\right) \leqslant \frac{64 c}{\varepsilon^{2}} \mathbf{E} q_{F}^{2}\left(\int_{\mathbf{R}} \varphi d F\right) .
$$

Now the key to our problem is to estimate $\mathbf{E} q_{F}^{2}\left(\int_{\mathbf{R}} \varphi d F\right)$. If we are able to prove that for any $\eta>0$, there is a finite dimensional closed subspace $F$ in $B$ such that for any $E \in \mathscr{R}$,

$$
\mathbf{E} q_{F}^{2}(F(E)) \leqslant c \eta \mu(E),
$$

then it is not hard to obtain

$$
\mathbf{E} q_{F}^{2}\left(\int_{\mathbf{R}} \varphi d F\right) \leqslant c \eta \int_{\mathbf{R}} \varphi^{2} d \mu=c \eta\|\varphi\|_{0}^{2} .
$$

Thus (3.14) can be proved by choosing $\eta$ small enough, i.e., taking a finite dimensional closed subspace $F$.

Let us return to the proof of (3.16). Since $\left\{F(E) / \mu^{1 / 2}(E), E \in \mathscr{R}\right\}$ is uniformly tight, then for any $\eta>0$ there exists a finite dimensional closed subspace $F$ such that

$$
\sup _{E} \mathbf{P}\left\{\omega: q_{F}^{2}(F(E))>\eta \mu(E)\right\}<\eta^{2} .
$$

Moreover, we have

$$
\begin{aligned}
\mathbf{E} q_{F}^{2}(F(E)) & \leqslant \eta \mu(E)+\int_{\left\{\omega: q_{F}^{2}(F(E))>\eta \mu(E)\right\}} q_{F}^{2}(F(E)) d \mathbf{P} \\
& \leqslant \eta \mu(E)+\left(\mathbf{E}\|F(E)\|^{4}\right)^{1 / 2}\left(\mathbf{P}\left\{\omega: q_{F}^{2}(F(E))>\eta \mu(E)\right\}\right)^{1 / 2} \\
& \leqslant \eta \mu(E)+c \mu(E) \eta \leqslant(1+c) \eta \mu(E) .
\end{aligned}
$$

After having the previous argument, we can now prove (3.12). In fact, we have in a similar way to the proof of (3.10)

$$
\begin{aligned}
& \mathbf{P}\left\{\omega: \sup _{\|\varphi\|_{q} \leqslant 1} q_{F}^{2}\left(X_{n}(\varphi)\right)>\dot{\varepsilon}\right\} \leqslant \frac{e}{e-1} \mathbf{E}\left(1-e^{-\varepsilon^{-1} \sup _{\|\varphi\|_{q} \leqslant 1} q_{F}^{2}\left(X_{n}\left(\varphi_{N}\right)\right)}\right) \\
& \quad \leqslant \frac{e}{e-1} \lim _{N \rightarrow \infty} \mathbf{E} \sup _{f \in F^{\perp}} \operatorname{Re}\left(1-e^{i\left\langle f, X_{n}\left(\Phi_{N}\right)\right\rangle}\right),
\end{aligned}
$$

where

$$
\Phi_{N}=\sum_{j=1}^{N} Y_{j} e_{j}, \quad\left\{Y_{j}, j \geqslant 1\right\}
$$


is a sequence of i.i.d. $N(0,2 / \varepsilon),\left\{e_{j}\right\}$ is a CONS in $\mathscr{S}$ relative to $\|\cdot\|_{q}$, and $F^{\perp}$ is the annihilator of $F$.

And the limit term of (3.18) can be estimated as follows:

$$
\begin{aligned}
\mathbf{E} & \sup _{f \in F^{\perp}} \operatorname{Re}\left(1-e^{i\left\langle f, X_{n}\left(\Phi_{N}\right)\right\rangle}\right)=\mathbf{E} \sup _{f \in F^{\perp}} \operatorname{Re}\left(1-e^{i\left\langle f, X_{n}\left(\Phi_{N}\right)\right\rangle}\right) I_{\left(\left\|\Phi_{N}\right\|_{p} \leqslant 4 C / \varepsilon\right)} \\
& +\mathbf{E} \sup _{f \in F^{\perp}} \operatorname{Re}\left(1-e^{i\left\langle f, X_{n}\left(\Phi_{N}\right)\right\rangle}\right) I_{\left(\left\|\Phi_{N}\right\|_{p}>4 C / \varepsilon\right)} \\
\leqslant & 2 \mathbf{E}\left(q_{F}\left(X_{n}\left(\Phi_{N}\right)\right) \wedge 1\right) I_{\left(\left\|\varphi_{N}\right\|_{p} \leqslant 4 C / \varepsilon\right)}+2 \mathbf{P}\left\{\omega:\left\|\varphi_{N}\right\|_{p}>\frac{4 C}{\varepsilon}\right\} \\
\leqslant & 2 \sup _{\|\varphi\|_{p} \leqslant 4 C / \varepsilon} \mathbf{E}\left(q_{F}\left(X_{n}(\varphi)\right) \wedge 1\right)+4 \mathbf{P}\left\{\omega:\left\|\sum_{j=1}^{\infty} Y_{j} e_{j}\right\|>\frac{4 C}{\varepsilon}\right\} \\
< & 2\left(\frac{\varepsilon}{8}+\sup _{\|\varphi\|_{p} \leqslant 4 C / \varepsilon} \mathbf{P}\left\{\omega: q_{F}\left(X_{n}(\varphi)\right)>\frac{\varepsilon}{8}\right\}\right) \\
& +4 \mathbf{P}\left\{\omega:\left\|\sum_{j=1}^{\infty} Y_{j} e_{j}\right\|>\frac{4 C}{\varepsilon}\right\}<\varepsilon .
\end{aligned}
$$

Up to now, we have completed the key step (i), i.e., $X_{n}$ is uniformly tight in $\mathscr{L}_{b}(\mathscr{S}, B)$. Next we shall go on to prove (ii).

For this let us recall some preliminary results concerning the integral representation formula of dual $\mathscr{L}_{b}^{\prime}(\mathscr{S}, B)$ of $\mathscr{L}_{b}(\mathscr{S}, B)$ (see [9] for details). Let $\mathscr{B}\left(\mathscr{S}^{\prime}, B\right)$ denote the space of continuous bilinear forms on $\mathscr{S}^{\prime} \times B$ and carry the topology of uniform convergence on the products of bounded sets, $\mathscr{S}^{\prime} \hat{\otimes} B$ denote the projective tensor product of $\mathscr{S}^{\prime}$ and $B$. Since $\mathscr{S}$ and $\mathscr{S}^{\prime}$ are nuclear spaces, then we have the canonical isomorphism

$$
\mathscr{S}^{\prime} \hat{\otimes} B \cong \mathscr{L}_{b}(\mathscr{S}, B), \quad\left(\mathscr{S}^{\prime} \hat{\otimes} B\right)^{\prime} \cong \mathscr{B}\left(\mathscr{S}^{\prime}, B\right),
$$

where the dual $\left(\mathscr{S}^{\prime} \hat{\otimes} B\right)^{\prime}$ carries the strong dual topology. The following lemma is Proposition 49.1 in [9].

Lemma 3.1. $A$ bilinear form $u$ on $\mathscr{S}^{\prime} \times B$ is continuous if and only if there is a weakly closed equicontinuous subset $A$ (respectively $M^{\prime}$ ) of $\mathscr{S}$ (respectively $B^{\prime}$ ) and a positive Radon measure $v$ on the compact set $A \times M^{\prime}$ with the total mass $\leqslant 1$, such that for all $\varphi^{\prime} \in \mathscr{S}^{\prime}, x \in B$,

$$
u\left(\varphi^{\prime}, x\right)=\int_{A \times M^{\prime}}\left\langle\varphi^{\prime}, \varphi\right\rangle\left\langle x^{\prime}, x\right\rangle d v\left(\varphi, x^{\prime}\right) .
$$

For any given $f \in \mathscr{L}_{b}^{\prime}(\mathscr{S}, B)$ and $T_{F} \in \mathscr{L}_{b}(\mathscr{S}, B)$ there exist $\varphi_{F}^{\prime} \in \mathscr{S}^{\prime}$ and $x_{F} \in B$ such that $T_{F}$ is the image of $\varphi_{F}^{\prime} \otimes x_{F}$ under the canonical isomophism mapping, and further there is a $\psi_{f} \in \mathscr{B}(\mathscr{S}, B)$ such that $\psi_{f}\left(\varphi_{F}^{\prime}, x_{F}\right)=f\left(T_{F}\right)$. From this and Lemma 3.1 we derive that there are a weakly closed equicontinuous subset $A$ (respectively $M^{\prime}$ ) of $\mathscr{S}$ (respectively $B^{\prime}$ ) and a positive Radon measure $v$ on the compact set $A \times M^{\prime}$ with the 
total mass $\leqslant 1$ such that

$$
\begin{aligned}
f\left(T_{F}\right) & =\psi_{f}\left(\varphi_{F}^{\prime}, x_{F}\right)=\int_{A \times M^{\prime}}\left\langle\varphi_{F}^{\prime}, \varphi\right\rangle\left\langle x^{\prime}, x_{F}\right\rangle d v\left(\varphi, x^{\prime}\right) \\
& =\int_{A \times M^{\prime}}\left\langle x^{\prime}, T_{F}(\varphi)\right\rangle d v\left(\varphi, x^{\prime}\right) .
\end{aligned}
$$

Thus we have

$$
\begin{aligned}
\mathbf{E} f^{2}\left(T_{F}\right) & =\mathbf{E}\left(\int_{A \times M^{\prime}}\left\langle x^{\prime}, T_{F}(\varphi)\right\rangle d v\left(\varphi, x^{\prime}\right)\right)^{2} \\
& \leqslant \int_{A \times M^{\prime}} \mathbf{E}\left\langle x^{\prime}, T_{F}(\varphi)\right\rangle^{2} d v\left(\varphi, x^{\prime}\right) \\
& \leqslant \int_{A \times M^{\prime}}\left\|x^{\prime}\right\|^{2} \mathbf{E}\left\|T_{F}(\varphi)\right\|^{2} d v\left(\varphi, x^{\prime}\right) \\
& \leqslant c \int_{A \times M^{\prime}}\left\|x^{\prime}\right\|^{2}\|\varphi\|_{0}^{2} d v\left(\varphi, x^{\prime}\right) .
\end{aligned}
$$

In addition, since $A$ and $M^{\prime}$ are weakly compact in $\mathscr{S}$ and $B^{\prime}$ respectively, then $A$ and $M^{\prime}$ are weakly bounded, and hence $A$ is bounded with respect to the semi-norms $\|\cdot\|_{p}, 0 \leqslant p<\infty$, and $M^{\prime}$ is bounded with respect to the dual norm. This, together with (3.23), implies that $\mathbf{E} f^{2}\left(T_{F}\right)<\infty$. Thus (ii) is a direct consequence of the classical central limit theorem.

The proof of Theorem 3.1 is now completed.

4. The CLT for measure-valued processes arising from Brownian motions. Suppose that $\left\{X, X_{n}, n \geqslant 1\right\}$ is a sequence of i.i.d. symmetric $B$-valued random vectors, $\left\{B(t), B_{i}(t), i \geqslant 1,0 \leqslant t \leqslant 1\right\}$ a sequence of i.i.d. standard Brownian motions. Assume furthermore that they are independent of each other. Set

$$
Z_{n}(t)=\frac{1}{\sqrt{n}} \sum_{i=1}^{n} X_{i} \delta_{B_{i}(t)}, \quad 0 \leqslant t \leqslant 1 .
$$

This is a measure-valued stochastic process. As in Section 3 we shall consider $Z_{n}$ as a sequence of $\mathscr{L}_{b}(\mathscr{S}, B)$-valued continuous random processes on $[0,1]$. The reader is referred to the case having no weighted term $X_{i}$ in $Z_{n}$. To make our problem clearer, let us first see two special cases.

Theorem 4.1. Suppose that $B$ is the space of type $2,\left\{X, X_{i}, i \geqslant 1\right\}$ and $\left\{B(t), B_{i}(t), i \geqslant 1,0 \leqslant t \leqslant 1\right\}$ are as above with $\mathbf{E}\|X\|^{2}<\infty$. Define

$$
Z_{n}\left(t_{0}\right)=\frac{1}{\sqrt{n}} \sum_{i=1}^{n} X_{i} \delta_{B_{i}\left(t_{0}\right)}, \quad \text { for some } t_{0} \in[0,1] \text {. }
$$

Then $\left\{Z_{n}(t)(\varphi), \varphi \in \mathscr{S}\right\}$ converges weakly to a Gaussian process $G=$ $\{G(\varphi), \varphi \in \mathscr{S}\}$.

$\mathrm{Pr}$ o of. This in fact is a direct consequence of the proof of Theorem 3.1. Observe that in the proof of Theorem 3.1 the hypotheses that 
$\mathbf{E}\|F(E)\|^{4} \leqslant c \mu^{2}(E)$ for some constant $c>0$ and $\left\{F(E) / \mu^{1 / 2}(E), E \in \mathscr{R}\right\}$ is uniformly tight in $B$ are used only in (3.16).

But if letting $F(E)=X \delta_{B\left(t_{0}\right)}(E)$, then

$\mathbf{E} q_{F}^{2}(F(E))=\mathbf{E} q_{F}^{2}(X) \mathbf{E} \delta_{B\left(t_{0}\right)}(E)=\mathbf{E} q_{F}^{2}(X) \mathbf{P}\left\{\omega: B\left(t_{0}, \omega\right) \in E\right\}$.

Obviously, for any $\varepsilon>0$ there exists a finite dimensional closed subspace $F$ in $B$ such that $\mathbf{E} q_{F}^{2}(X)<\varepsilon$ whenever $\mathbf{E}\|X\|^{2}<\infty$. In addition, $\mathbf{P}\left\{\omega: B\left(t_{0}, \omega\right) \in E\right\} \leqslant c \mu(E)$ for some numerical constant $c>0$. These imply (3.16), and hence finish the proof of Theorem 4.1.

Theorem 4.2. Suppose that $B$ is the space of type $2,\left\{X, X_{i}, i \geqslant 1\right\}$ and $\left\{B(t), B_{i}(t), i \geqslant 1,0 \leqslant t \leqslant 1\right\}$ are as above with $\mathbf{E}\|X\|^{2}<\infty$. Define

$$
Z_{n}(\varphi)(t)=\frac{1}{\sqrt{n}} \sum_{i=1}^{n} X_{i} \varphi\left(B_{i}(t)\right), \quad 0 \leqslant t \leqslant 1, \quad \text { for some } \varphi \in \mathscr{S} .
$$

Then $\left\{Z_{n}(\varphi)(t), 0 \leqslant t \leqslant 1\right\}$ converges weakly to a Gaussian process $G_{\varphi}=$ $\left\{G_{\varphi}(t), 0 \leqslant t \leqslant 1\right\}$ in $C([0,1], B)$, the set of all bounded continuous mappings from $[0,1]$ into $B$ with the uniform norm topology.

$\mathrm{P}$ r o o f. It is clear that for two finite sequences $\left(t_{1}, t_{2}, \ldots, t_{m}\right)$ and $\left(f_{1}, f_{2}, \ldots, f_{m}\right)$ the $m$-dimensional random vector $\left(f_{1}(X) \varphi\left(B\left(t_{1}\right)\right), \ldots\right.$, $\left.f_{m}(X) \varphi\left(B\left(t_{m}\right)\right)\right)$ satisfies the classical central limit theorem. Also, it is easily seen that the class of all cylindrical sets having the form

$$
\left\{x \in C([0,1], B):\left(\left\langle f_{1}, x\left(t_{1}\right)\right\rangle, \ldots,\left\langle f_{m}, x\left(t_{m}\right)\right\rangle\right) \in A, A \in \mathscr{B}\left(\mathbf{R}^{m}\right)\right\}
$$

generates the Borel $\sigma$-field $\mathscr{B}(C([0,1], B))$. Thus we need only turn our attention to the relative compactness of $\left\{Z_{n}, n \geqslant 1\right\}$ in $C([0,1], B)$. By Theorem 4.4 in [4] and Lemma 2.2 in [6], this will be shown if

(i) $\left\{\left\langle f, Z_{n}(\varphi)(t)\right\rangle, t \in[0,1]\right\}$ is relatively compact in $C([0,1])$ for each $f \in B^{\prime}$,

(ii) for any $\varepsilon>0$ there exist a positive constant $M$ and a finite dimensional closed subspace $F$ in $B$ such that

$$
\begin{aligned}
& \sup _{n} \mathbf{P}\left\{\omega: \sup _{0 \leqslant t \leqslant 1}\left\|Z_{n}(\varphi)(t)\right\|>M\right\}<\varepsilon, \\
& \sup _{n} \mathbf{P}\left\{\omega: \sup _{0 \leqslant t \leqslant 1} q_{F}\left(Z_{n}(\varphi)(t)\right)>\varepsilon\right\}<\varepsilon .
\end{aligned}
$$

For (i) it is enough to verify that for any $\varepsilon>0$ there are $\delta>0$ and $M>0$ such that

$$
\begin{array}{r}
\sup _{n} \mathbf{P}\left\{\omega: \sup _{|t-s|<\delta}\left|\frac{1}{\sqrt{n}} \sum_{i=1}^{n} f\left(X_{i}\right)\left(\varphi\left(B_{i}(t)\right)-\varphi\left(B_{i}(s)\right)\right)\right|>\varepsilon\right\}<\varepsilon, \\
\sup _{n} \mathbf{P}\left\{\omega: \sup _{0 \leqslant t \leqslant 1}\left|\frac{1}{\sqrt{n}} \sum_{i=1}^{n} f\left(X_{i}\right) \varphi\left(B_{i}(t)\right)\right|>M\right\}<\varepsilon .
\end{array}
$$


Let us prove (4.6) and (4.7) by making use of the metric entropy techniques. In fact, if we denote

$$
D_{1}=\left\{\omega: \sum_{i=1}^{n} f^{2}\left(X_{i}\right) \leqslant a^{2} n\right\}
$$

we have

$$
\begin{aligned}
& \mathbf{P}\left\{\omega: \sup _{|t-s|<\delta}\left|\frac{1}{\sqrt{n}} \sum_{i=1}^{n} f\left(X_{i}\right)\left(\varphi\left(B_{i}(t)\right)-\varphi\left(B_{i}(s)\right)\right)\right|>\varepsilon\right\} \\
& \leqslant \mathbf{P}\left\{\omega: \sup _{|t-s|<\delta}\left|\frac{1}{\sqrt{n}} \sum_{i=1}^{n} f\left(X_{i}\right) I_{D_{1}}\left(\varphi\left(B_{i}(t)\right)-\varphi\left(B_{i}(s)\right)\right)\right|>\varepsilon\right\} \\
& \quad+\mathbf{P}\left(\bar{D}_{1}\right)
\end{aligned}
$$

and

$$
\begin{aligned}
& \mathbf{P}\left\{\omega: \sup _{0 \leqslant t \leqslant 1}\left|\frac{1}{\sqrt{n}} \sum_{i=1}^{n} f\left(X_{i}\right) \varphi\left(B_{i}(t)\right)\right|>M\right\} \\
& \quad \leqslant \mathbf{P}\left\{\omega: \sup _{0 \leqslant t \leqslant 1}\left|\frac{1}{\sqrt{n}} \sum_{i=1}^{n} f\left(X_{i}\right) I_{D_{1}} \varphi\left(B_{i}(t)\right)\right|>M\right\}+\mathbf{P}\left(\bar{D}_{1}\right),
\end{aligned}
$$

where $a, \delta$ and $M$ are to be specified later.

Taking $a^{2} \geqslant 2 \mathbf{E} f^{2}(X) / \varepsilon$, we have

$$
\sup _{n} \mathbf{P}\left\{\omega: \sum_{i=1}^{n} f^{2}\left(X_{i}\right) \geqslant a^{2} n\right\}<\frac{\varepsilon}{2} .
$$

On the other hand, set $\beta_{1}(\varphi)=\sup _{x \in \mathbf{R}}|d \varphi(x) / d x|<\infty$, then

$$
\left|\varphi\left(B_{i}(t)\right)-\varphi\left(B_{i}(s)\right)\right| \leqslant \beta_{1}(\varphi)\left|B_{i}(t)-B_{i}(s)\right|,
$$

and hence we obtain

$$
\begin{aligned}
& \mathbf{P}\left\{\omega:\left|\frac{1}{\sqrt{n}} \sum_{i=1}^{n} f\left(X_{i}\right) I_{D_{1}}\left(\varphi\left(B_{i}(t)\right)-\varphi\left(B_{i}(s)\right)\right)\right|>a \beta_{1}(\varphi)|t-s|^{1 / 2} u\right\} \\
& \quad=\mathbf{P}\left\{\left|\frac{1}{\sqrt{n}} \sum_{i=1}^{n} \varepsilon_{i} f\left(X_{i}\right) I_{D_{1}}\left(\varphi\left(B_{i}(t)\right)-\varphi\left(B_{i}(s)\right)\right)\right|>a \beta_{1}(\varphi)|t-s|^{1 / 2} u\right\} \\
& \quad \leqslant 2 \mathbf{E}_{X} \mathbf{P}\left\{\omega:\left|\frac{1}{\sqrt{n}} \sum_{i=1}^{n} \varepsilon_{i} f\left(X_{i}\right) I_{D_{1}} \frac{B_{i}(t)-B_{i}(s)}{|t-s|^{1 / 2}}\right|>a u\right\} \\
& \quad \leqslant 4 \mathbf{E}_{X} \exp \left(-\frac{u^{2} a^{2}}{\frac{2}{n} \sum_{i=1}^{n} f^{2}\left(X_{i}\right) I_{D_{1}}}\right) \leqslant 4 \exp \left(-\frac{u^{2}}{2}\right) .
\end{aligned}
$$

In this way, it is easy to see that

$$
\left\{\frac{(\sqrt{n})^{-1} \sum_{i=1}^{n} f\left(X_{i}\right) I_{D_{1}}\left(\varphi\left(B_{i}(t)\right)-\varphi\left(B_{i}(s)\right)\right)}{a \beta_{1}(\varphi)}, \quad t, s \in[0,1]\right\}
$$


is a sub-Gaussian process with the metric $d(s, t)=|t-s|^{1 / 2}$. By Theorem 11.6 in [6] we know that

$$
\begin{aligned}
& \sup _{n} \mathbf{E} \sup _{|t-s|<\delta}\left|\frac{1}{\sqrt{n}} \sum_{i=1}^{n} f\left(X_{i}\right) I_{D_{1}}\left(\varphi\left(B_{i}(t)\right)-\varphi\left(B_{i}(s)\right)\right)\right| \\
& \leqslant k a \beta_{1}(\varphi) \int_{0}^{\delta}(\ln N([0,1], d, \varepsilon))^{1 / 2} d \varepsilon, \\
& \mathbf{E} \sup _{0 \leqslant t \leqslant 1}\left|\frac{1}{\sqrt{n}} \sum_{i=1}^{n} f\left(X_{i}\right) I_{D_{1}} \varphi\left(B_{i}(t)\right)\right| \\
& \leqslant k a \beta_{1}(\varphi) \int_{0}^{1}(\ln N([0,1], d, \varepsilon))^{1 / 2} d \varepsilon<\infty,
\end{aligned}
$$

where $k$ is an absolute constant and $N([0,1], d, \varepsilon)$ is the smallest number of balls of radius $\varepsilon$ in the metric $d$ which covers $[0,1]$.

Now we can choose $\delta$ so small that (4.6) holds and $M$ so large that (4.7) holds. Thus the proof of (i) is concluded.

It remains to prove (ii). The idea of its proof is similar to that used above, i.e., we can still apply the metric entropy techniques to some appropriate vector-valued sub-Gaussian processes.

Set $\psi_{2}(x)=\exp x^{2}-1,\|\cdot\|_{\psi_{2}(d \mathbf{P})}$ denote its Orlicz norm with respect to probability space $(\Omega, \mathscr{F}, \mathbf{P})$. We only prove (4.5) since the proof of (4.4) is similar and simpler. By the well-known contractive principle we have

$$
\begin{array}{r}
\mathbf{E} \exp \left(\frac{1}{C^{2}} q_{F}^{2}\left(\frac{1}{\sqrt{n}} \sum_{i=1}^{n} X_{i} I_{D_{2}}\left(\varphi\left(B_{i}(t)\right)-\varphi\left(B_{i}(s)\right)\right)\right)\right) \\
\leqslant \mathbf{E} \exp \left(\frac{\beta_{1}^{2}(\varphi)}{C^{2}} q_{F}^{2}\left(\frac{1}{\sqrt{n}} \sum_{i=1}^{n} X_{i} I_{D_{2}}\left(B_{i}(t)-B_{i}(s)\right)\right)\right),
\end{array}
$$

where $C>0$ is arbitrary, $a$ and $F$ are to be specified below and $D_{2}=$ $\left\{\omega: \sum_{i=1}^{n} q_{F}^{2}\left(X_{i}\right) \leqslant a^{2} n\right\}$. Then

$$
\begin{aligned}
& \left\|q_{F}\left(\frac{1}{\sqrt{n}} \sum_{i=1}^{n} X_{i} I_{D_{2}}\left(\varphi\left(B_{i}(t)\right)-\varphi\left(B_{i}(s)\right)\right)\right)\right\|_{\psi_{2}(d \mathbf{P})} \\
& \leqslant \beta_{1}(\varphi)\left\|q_{F}\left(\frac{1}{\sqrt{n}} \sum_{i=1}^{n} X_{i} I_{D_{2}}\left(B_{i}(t)-B_{i}(s)\right)\right)\right\|_{\psi_{2}(d \mathbf{P})} \\
& \quad \leqslant c \beta_{1}(\varphi) a|t-s|^{1 / 2} .
\end{aligned}
$$

From the above and noting Remark 11.5 in [6] we deduce

$$
\begin{aligned}
& \mathbf{E} \sup _{0 \leqslant t \leqslant 1} q_{F}\left(\frac{1}{\sqrt{n}} \sum_{i=1}^{n} X_{i} I_{D_{2}}\left(\varphi\left(B_{i}(t)\right)-\varphi\left(B_{i}(s)\right)\right)\right) \\
& \leqslant c \beta_{1}(\varphi) a \int_{0}^{1}(\ln N([0,1], d, \varepsilon))^{1 / 2} d \varepsilon
\end{aligned}
$$


After taking $a>0$ so small that $c \beta_{1}(\varphi) a \int_{0}^{1}(\ln N([0,1], d, \varepsilon))^{1 / 2} d \varepsilon<$ $\varepsilon / 2$, we can choose a finite dimensional closed subspace $F$ in $B$ such that $\mathbf{E} q_{F}^{2}(X) / a^{2}<\varepsilon / 2$. Moreover, we have

$$
\begin{aligned}
& \mathbf{P}\left\{\omega: \sup _{0 \leqslant t \leqslant 1} q_{F}\left(\frac{1}{\sqrt{n}} \sum_{i=1}^{n} X_{i} \varphi\left(B_{i}(t)\right)\right)>\varepsilon\right\} \\
& \quad \leqslant \mathbf{P}\left\{\omega: \sup _{0 \leqslant t \leqslant 1} q_{F}\left(\frac{1}{\sqrt{n}} \sum_{i=1}^{n} X_{i} I_{D_{2}} \varphi\left(B_{i}(t)\right)\right)>\varepsilon\right\}+\mathbf{P}\left(\bar{D}_{2}\right)<\varepsilon .
\end{aligned}
$$

Up to now we have showed (ii), and hence Theorem 4.2.

At the end of this paper we shall give the following Theorem 4.3, which motivates the present work.

Theorem 4.3. Suppose that $B$ is the space of type $2,\left\{X, X_{i}, i \geqslant 1\right\}$ and $\left\{B(t), B_{i}(t), i \geqslant 1,0 \leqslant t \leqslant 1\right\}$ are as above with $\mathbf{E}\|X\|^{2}<\infty$. Define

$$
Z_{n}(\varphi)(t)=\frac{1}{\sqrt{n}} \sum_{i=1}^{n} X_{i} \varphi\left(B_{i}(t)\right), \quad 0 \leqslant t \leqslant 1, \varphi \in \mathscr{S} .
$$

Then $Z_{n}$ converges weakly to a Gaussian process $G$ in $C\left([0,1], \mathscr{L}_{b}(\mathscr{S}, B)\right)$, equipped with the locally convex topology generated by a family of semi-norms.

The proof of Theorem 4.3 is basically along the lines given in [7] and [10], some necessary changes can be made according to Theorem 3.1. For reader's convenience, we give some key points below.

Proposition 4.1. If $K$ is compact in $C\left([0,1], \mathscr{L}_{b}(\mathscr{S}, B)\right)$ then there exists a $p \in \mathbf{N}$ such that $K$ is also compact in $C\left([0,1], \mathscr{L}_{b}\left(\mathscr{S}_{p}, B\right)\right)$.

P r o of. For each $\varphi$ in $\mathscr{S}$ the set $\{x(\varphi), x \in K\}$ is compact in $C([0,1], B)$, then the following properties hold: $\{x(\varphi)(t), 0 \leqslant t \leqslant 1, x \in$ $K\}$ is relatively compact in $B$ and

$$
\limsup _{\delta \rightarrow 0} \sup _{x \in K}\|x(\varphi)(t)-x(\varphi)(s)\|=0 .
$$

Since $\sup _{x \in K} \sup _{0 \leqslant t \leqslant 1}\|x(\varphi)(t)\|<\infty$, then the Banach-Steinhaus theorem shows that there exist $q \in \mathbf{N}$ and $L>0$ such that

$$
\sup _{x \in K} \sup _{0 \leqslant t \leqslant 1}\|x(\varphi)(t)\| \leqslant L\|\varphi\|_{q} .
$$

Since $\mathscr{S}$ is nuclear, there are a natural number $r>q$ and a CONS $\left(e_{j}\right)$ relative to $\mathscr{S}_{r}$ such that $\sum_{j=1}^{\infty}\left\|e_{j}\right\|_{q}^{2}<\infty$, so that we have

$$
\begin{aligned}
& \sup _{x \in K} \sup _{0 \leqslant t \leqslant 1} \sup _{\|\varphi\|_{r} \leqslant 1}\|x(\varphi)(t)\|^{2}=\sup _{x \in K} \sup _{0 \leqslant t \leqslant 1} \sup _{\|\varphi\|_{r} \leqslant 1} \sup _{f \in B_{1}^{\prime}}|\langle f, x(\varphi)(t)\rangle|^{2} \\
& =\sup _{x \in K} \sup _{0 \leqslant t \leqslant 1} \sup _{f \in B_{1}^{\prime}} \sum_{j=1}^{\infty}\left\langle f, x\left(e_{j}\right)(t)\right\rangle^{2} \\
& \quad \leqslant \sup _{x \in K} \sup _{0 \leqslant t \leqslant 1} \sup _{f \in B_{1}^{\prime}} \sum_{j=1}^{\infty}\|f\|^{2}\left\|x\left(e_{j}\right)(t)\right\|^{2}<\infty
\end{aligned}
$$


Since $\sup _{x \in K} \sup _{|t-s|<\delta}\left\|x\left(e_{j}\right)(t)-x\left(e_{j}\right)(s)\right\|^{2} \leqslant 4 L^{2}\left\|e_{j}\right\|_{q}^{2}$, then by the Lebesgue convergence theorem and (4.17) we get

$$
\begin{aligned}
& \limsup _{\delta \rightarrow 0} \sup _{x \in K} \sup _{|t-s|<\delta}\|x(\varphi)(t)-x(\varphi)(s)\| \\
& \quad=\lim _{\delta \rightarrow 0} \sup _{x \in K} \sup _{|t-s|<\delta} \sup _{f \in B_{1}^{\prime}} \sup _{\|\varphi\|_{r} \leqslant 1}\langle f, x(\varphi)(t)-x(\varphi)(s)\rangle \\
& \quad \leqslant \lim _{\delta \rightarrow 0} \sup _{x \in K} \sup _{|t-s|<\delta} \sup _{f \in B_{1}^{\prime}}\left(\sum_{j=1}^{\infty}\left\langle f, x\left(e_{j}\right)(t)-x\left(e_{j}\right)(s)\right\rangle^{2}\right)^{1 / 2} \\
& \quad \leqslant\left(\sum_{j=1}^{\infty} \lim _{\delta \rightarrow 0} \sup _{x \in K} \sup _{|t-s|<\delta}\left\|x\left(e_{j}\right)(t)-x\left(e_{j}\right)(s)\right\|^{2}\right)^{1 / 2}=0 .
\end{aligned}
$$

On the other hand, since $\mathscr{S}$ is nuclear, there exist a natural number $p>r$ and a CONS $\left(e_{j}\right)$ relative to $\mathscr{S}_{p}$ such that

$$
\sum_{j=1}^{\infty}\left\|e_{j}\right\|_{r}^{2}<\infty
$$

Then it follows from (4.19) that

$$
\lim _{N \rightarrow \infty} \sum_{j=N}^{\infty} \sup _{x \in K} \sup _{0 \leqslant t \leqslant 1}\left\|\left\langle x(t), e_{j}\right\rangle\right\|^{2}=0,
$$

so that $\{x(t), x \in K\}$ is relatively compact in $\mathscr{L}_{b}\left(\mathscr{S}_{p}, B\right)$ for each $0 \leqslant t \leqslant 1$.

Since $\|\cdot\|_{-r} \geqslant\|\cdot\|_{-p}$, by (4.20) we have

$$
\limsup _{\delta \rightarrow 0} \sup _{x \in K} \sup _{|t-s|<\delta}\|x(\varphi)(t)-x(\varphi)(s)\|=0 .
$$

Thus $K$ has compact closure in $C\left([0,1], \mathscr{L}_{b}\left(\mathscr{S}_{p}, B\right)\right)$. And $K$ is automatically closed in $C\left([0,1], \mathscr{L}_{b}\left(\mathscr{S}_{p}, B\right)\right)$ by the definition of the topology on $C\left([0,1], \mathscr{L}_{b}\left(\mathscr{S}_{p}, B\right)\right)$. The proof of Proposition 4.1 is completed.

Proposition 4.1 will enable us to use Lemma 2.1. Now for each $\varepsilon>0$ we try to prove the following two claims:

(i) there exist $p \in \mathbf{N}$ and $M>0$ such that

$$
\sup _{n} \mathbf{P}\left\{\omega: \sup _{0 \leqslant t \leqslant 1} \sup _{\|\varphi\|_{p} \leqslant 1}\left\|Z_{n}(\varphi)(t)\right\|>M\right\}<\frac{\varepsilon}{4},
$$

(ii) there exists a finite dimensional closed subspace $F$ in $B$ such that

$$
\sup _{n} \mathbf{P}\left\{\omega: \sup _{0 \leqslant t \leqslant 1} \sup _{\|\varphi\|_{q} \leqslant 1} q_{F}\left(Z_{n}(\varphi)(t)\right)>\varepsilon\right\}<\frac{\varepsilon}{4},
$$

where $q$ is such that the natural embedding $i: \mathscr{S}_{q} \rightarrow \mathscr{S}_{p}$ is nuclear.

The claim (i) can be proved in a completely similar way to (3.5). Before the proof of (ii) is given in details, let us see how we shall verify the relative compactness of $\left\{Z_{n}, n \geqslant 1\right\}$ in $C\left([0,1], \mathscr{L}_{b}(\mathscr{S}, B)\right)$. Given any $\varepsilon>0$, 
$p$ and $q$ are taken by (i) and (ii). Let $\left(e_{j}\right)$ be a CONS in $\mathscr{S}_{q}$, for each $e_{j}$, choose $K_{j} \subset C\left([0,1], \mathscr{L}_{b}(\mathscr{S}, B)\right)$ such that

$$
\begin{gathered}
\sup _{n} \mathbf{P}\left\{\omega: Z_{n}\left(e_{j}\right) \in K_{j}\right\}>1-\frac{\varepsilon}{2^{j+1}}, \\
\lim \sup _{\delta \rightarrow 0} \sup _{x \in K_{j}} \sup _{|t-s|<\delta}\left\|Z_{n}\left(e_{j}\right)(t)-Z_{n}\left(e_{j}\right)(s)\right\|=0 .
\end{gathered}
$$

Now define

$$
\begin{aligned}
& K=\left\{x: \sup _{0 \leqslant t \leqslant 1\|\varphi\|_{p} \leqslant 1} \sup _{0}\|x(\varphi)(t)\| \leqslant M\right\} \\
& \cap\left\{x:\left\{x(\varphi)(t), 0 \leqslant t \leqslant 1,\|\varphi\|_{q} \leqslant 1\right\} \text { is relatively compact in } B\right\} \bigcap_{j=1}^{\infty} K_{j} .
\end{aligned}
$$

Thus $\sup _{n} \mathbf{P}\left\{\omega: Z_{n} \notin K\right\}<\varepsilon$ and $K$ has compact closure in $C\left([0,1], \mathscr{L}_{b}\left(\mathscr{S}_{p}, B\right)\right)$ for some $p>r$.

Since the injection of $C\left([0,1], \mathscr{L}_{b}\left(\mathscr{S}_{p}, B\right)\right)$ into $C\left([0,1], \mathscr{L}_{b}(\mathscr{S}, B)\right)$ is continuous, the closure of $K$ in $C\left([0,1], \mathscr{L}_{b}\left(\mathscr{S}_{p}, B\right)\right)$ is compact in $C\left([0,1], \mathscr{L}_{b}(\mathscr{S}, B)\right)$. All these arguments show that $\left\{Z_{n}, n \geqslant 1\right\}$ is relatively compact in $C\left([0,1], \mathscr{L}_{b}(\mathscr{S}, B)\right)$.

We should now return to the proof of (ii). As in Step 2 in the proof of Theorem 3.1 it is enough to show that for any $\varepsilon>0$ and $0<C<\infty$ there exist a positive constant $M$ and a finite dimensional closed subspace $F$ in $B$ such that

$$
\begin{aligned}
& \sup _{n} \mathbf{P}\left\{\omega: \sup _{0 \leqslant t \leqslant 1}\left\|Z_{n}(\varphi)(t)\right\|>M\right\}<\varepsilon, \quad \text { for all }\|\varphi\|_{p} \leqslant C<\infty, \\
& \sup _{n} \mathbf{P}\left\{\omega: \sup _{0 \leqslant t \leqslant 1} q_{F}\left(Z_{n}(\varphi)(t)\right)>\varepsilon\right\}<\varepsilon, \quad \text { for all }\|\varphi\|_{p} \leqslant C<\infty .
\end{aligned}
$$

We will only prove (4.24). This statement is slightly different from (4.5). The interested reader may make some comparisons to understand the point of these proofs better. In fact, set

$$
\begin{aligned}
d_{0}(s, t) & =|t-s|^{1 / 2} \\
d(s, t) & =\int_{0}^{d_{0}(s, t)}\left(\ln N\left([0,1], d_{0}, \varepsilon\right)\right)^{1 / 2} d \varepsilon \approx(|t-s| \ln |t-s|)^{1 / 2} .
\end{aligned}
$$

Then for $D_{3}=\left\{\omega: \sum_{i=1}^{n} q_{F}^{2}\left(X_{i}\right) \sup _{t \neq s}\left[\left(B_{i}(t)-B_{i}(s)\right) / d(s, t)\right] \leqslant a^{2} n\right\}$

$$
\begin{aligned}
& \mathbf{P}\left\{\omega: \sup _{0 \leqslant t \leqslant 1} q_{F}\left(Z_{n}(\varphi)(t)\right)>\varepsilon\right\} \\
& \quad \leqslant \mathbf{P}\left\{\omega: q_{F}\left(\frac{1}{\sqrt{n}} \sum_{i=1}^{n} \varepsilon_{i} X_{i} \varphi\left(B_{i}(t)\right) I_{D_{3}}\right)>\varepsilon\right\}+\mathbf{P}\left(\bar{D}_{3}\right)=: I_{1}+I_{2},
\end{aligned}
$$

where $a>0$ and $F \subset B$ will be specified later. 
To estimate $I_{1}$, observe that

$$
\begin{aligned}
& \mathbf{E}_{\varepsilon} q_{F}^{2}\left(\frac{1}{\sqrt{n}} \sum_{i=1}^{n} \varepsilon_{i} X_{i} I_{D_{3}}\left(\varphi\left(B_{i}(t)\right)-\varphi\left(B_{i}(s)\right)\right)\right) \\
& \quad \leqslant c \frac{1}{n} \sum_{i=1}^{n} q_{F}^{2}\left(X_{i}\right)\left(\varphi\left(B_{i}(t)\right)-\varphi\left(B_{i}(s)\right)\right)^{2} I_{D_{3}} \\
& \quad \leqslant c \frac{1}{n} \sum_{i=1}^{n} q_{F}^{2}\left(X_{i}\right)\left(\int_{B_{i}(s)}^{B_{i}(t)} \varphi^{\prime}(x) d x\right)^{2} I_{D_{3}} \\
& \quad \leqslant c \frac{1}{n} \sum_{i=1}^{n} q_{F}^{2}\left(X_{i}\right)\left[\int_{\mathbf{R}}\left|\varphi^{\prime}(x)\right|^{2} d x\right]\left|B_{i}(t)-B_{i}(s)\right| I_{D_{3}} \\
& \quad \leqslant \operatorname{cad}(s, t)\left\|\varphi^{\prime}\right\|_{0}^{2} \leqslant c a d(s, t),
\end{aligned}
$$

where we have used $\left\|\varphi^{\prime}\right\|_{0} \leqslant\|\varphi\|_{1}$ and $\|\varphi\|_{p} \leqslant C$.

Thus it is not hard to get

$$
\| q_{F}\left(\frac{1}{\sqrt{n}} \sum_{i=1}^{n} X_{i} I_{D_{3}} \cdot\left(\varphi\left(B_{i}(t)\right)-\varphi\left(B_{i}(s)\right)\right) \|_{\psi_{2}(d \mathbf{P})} \leqslant c a d(s, t) .\right.
$$

Since $\int_{0}^{1}(\ln N([0,1], d, \varepsilon))^{1 / 2} d \varepsilon<\infty$, then by taking $a$ (depending on $C$ and $\varepsilon>0$ ) small enough we have

$$
\begin{aligned}
& \mathbf{E} \sup _{0 \leqslant t \leqslant 1} q_{F}\left(\frac{1}{\sqrt{n}} \sum_{i=1}^{n} X_{i} I_{D_{3}} \varphi\left(B_{i}(t)\right)\right) \\
& \leqslant c a \int_{0}^{1}(\ln N([0,1], d, \varepsilon))^{1 / 2} d \varepsilon<\frac{\varepsilon^{2}}{2} .
\end{aligned}
$$

This implies $I_{1}<\varepsilon / 2$.

For the estimation of $I_{2}$, we give the following result concerning Brownian motion.

Lemma 4.1. $\mathbf{E} \sup _{t \neq s, 0 \leqslant t, s \leqslant 1}[(B(t)-B(s)) / d(s, t)]<\infty$.

P r o of. Let $\psi_{2}(x)=\exp x^{2}-1$, and $\|\cdot\|_{\psi_{2}(d \mathbf{P})}$ denote the Orlicz norm of a random variable with respect to probability space $(\Omega, \mathscr{F}, \mathbf{P})$ and $\|\cdot\|_{\psi(d \mu \times d \mu)}$ the Orlicz norm of a measurable function with respect to Lebesgue measure space $([0,1] \times[0,1], \mathscr{B}([0,1]) \times \mathscr{B}([0,1]), \mu \times \mu)$. According to main results in [3], we have $\sup _{t \neq s}|B(t)-B(s)| / d(s, t) \leqslant c Y(\omega)$, where $Y(\omega)=\left\|I_{\left(d_{0}(s, t) \neq 0\right)}\left[(B(t)-B(s)) / d_{0}(s, t)\right]\right\|_{\psi_{2}(d \mu \times d \mu)}$. So it is enough to show $\mathbf{E} Y(\omega)<\infty$. In fact, set $M=\sup _{t \neq s}\left\|(B(t)-B(s)) / d_{0}(s, t)\right\|_{\psi_{2}(d \mathbf{P})}<\infty$, then

$$
\begin{aligned}
\mathbf{P} & \{\omega: Y(\omega)>u\} \\
& \leqslant \mathbf{P}\left\{\omega: \int_{[0,1] \times[0,1]} \exp \left(\frac{|B(t)-B(s)|^{2}}{u^{2} d_{0}^{2}(s, t)}\right) I_{\left(d_{0}(s, t) \neq 0\right)} d \mu(t) d \mu(s)>2\right\} \\
& \leqslant \mathbf{P}\left\{\omega: \int_{[0,1] \times[0,1]} \exp \left(\frac{|B(t)-B(s)|^{2}}{M^{2} d_{0}^{2}(s, t)}\right) I_{\left(d_{0}(s, t) \neq 0\right)} d \mu(t) d \mu(s)>2^{u^{2} / M^{2}}\right\} \\
& \leqslant 2^{-u^{2} / M^{2}}
\end{aligned}
$$


from which the required result holds. Furthermore, $Y(\omega)$ is exponentially square integrable although we will not use this fact.

Now $I_{2}$ can be estimated as follows

$$
\begin{aligned}
I_{2} & \leqslant \frac{\mathbf{E} \sum_{i=1}^{n} q_{F}^{2}\left(X_{i}\right) \sup _{s \neq t}(|B(t)-B(s)| / d(s, t))}{a^{2} n} \\
& =\frac{1}{a^{2}} \mathbf{E} q_{F}^{2}(X) \mathbf{E} \sup _{s \neq t} \frac{|B(t)-B(s)|}{d(s, t)} .
\end{aligned}
$$

Since $\mathbf{E}\|X\|^{2}<\infty$ and $\mathbf{E} \sup _{s \neq t}(|B(t)-B(s)| / d(s, t))<\infty$, then for any $\varepsilon>0$, after $a$ is determined to satisfy $I_{1}<\varepsilon / 2$, we are able to choose a finite dimensional closed subspace $F$ in $B$ such that $I_{2}<\varepsilon$.

Summarizing the preceding arguments, we have shown that $\left\{Z_{n}, n \geqslant 1\right\}$ is relatively compact in $C\left([0,1], \mathscr{L}_{b}(\mathscr{S}, B)\right)$.

Finally, it remains to verify that for each $f$ in $C^{\prime}\left([0,1], \mathscr{L}_{b}(\mathscr{S}, B)\right)$, $\left\langle f, Z_{n}\right\rangle$ converges weakly to a normal random variable as in Theorem 3.1 and Theorem 4.2. The proof of Theorem 4.3 is completed.

Acknowledgments. This paper is one part of the author's $\mathrm{Ph}$. D. dissertation submitted to Fudan University at Shanghai. The author would like to express his gratitude to Professors Z. Y. Lin and C. R. Lu for their guidance and encouragement. The author also thanks Professor X. Fernique who kindly communicates him preprints. Many thanks are due to the anonymous referee for a careful reading and valuable suggestions.

\section{REFERENCES}

1. de Araujo A., Gine E. The Central Limit Theorem for Real and Banach Valued Random Variables. New York: Wiley, 1980, 223 p.

2. Dunford N., Schwartz J.T. Linear Operators. I: General Theory. New York: Wiley, $1988,858 \mathrm{p}$.

3. Fernique $X$. Regularité des trajectoires des fonctions aléatoires gaussiennes. - Lecture Notes in Math., 1975, v. 480, p. 1-96.

4. Fernique $X$. Convergence en loi de fonctions aléatoires continues ou càdlàg, propriétés de compacité des lois. - Lecture Notes in Math., 1991, v. 1485, p. 178-195.

5. Itô $K$. Distribution-valued processes arising from independent Brownian motions. Math. Z., 1983, v. 182, p. 17-33.

6. Ledoux M., Talagrand M. Probability in Banach Spaces. Berlin-Heidelberg: SpringerVerlag, 1991, $480 \mathrm{p}$.

7. Mitoma $I$. Tightness of probabilities on $C\left([0,1], \mathscr{S}^{\prime}\right)$ and $D\left([0,1], \mathscr{S}^{\prime}\right)$. - Ann. Probab., 1983, v. 11, p. 989-999.

8. Thang D. H. On the convergence of vector random measures. - Probab. Theory Relat. Fields, 1991 , v. 88, p. 1-16.

9. Treves $F$. Topological Vector Spaces, Distributions and Kernels. New York-London: Academic Press, 1967, 565 p.

10. Walsh J.B. An introduction to stochastic partial differential equations. - Lecture Notes in Math., 1986; v. 1180 , p. 265-437. 